\title{
Erratum: Guiding Spin Spirals by Local Uniaxial Strain Relief [Phys. Rev. Lett. 116, 017201 (2016)]
}

\author{
Pin-Jui Hsu, Aurore Finco, Lorenz Schmidt, André Kubetzka, Kirsten von Bergmann, and Roland Wiesendanger \\ (Received 17 April 2018; published 14 May 2018)
}

DOI: 10.1103/PhysRevLett.120.209901

We have realized that in the published Fig. 1(d) the rectangles and arrows, which indicate the unit cells and crystallographic directions, were not in the correct positions. In addition, some of the scale bars in the published scanning tunneling microscopy images were incorrect. For Fig. 2 this leads to slightly different values for the dislocation line distance of $4.2 \mathrm{~nm}$ (instead of the $4.7 \mathrm{~nm}$ given in the published caption) and the magnetic period of now $1.5 \mathrm{~nm}$ (instead of $1.6 \mathrm{~nm}$ as mentioned in the published manuscript on page 3, left column, line 5). We are presenting here the revised Figs. 1, 2, and 3. Except for the roughly $10 \%$ change of the dislocation line distance and magnetic period of Fig. 2, this Erratum does not affect the conclusions of the Letter.

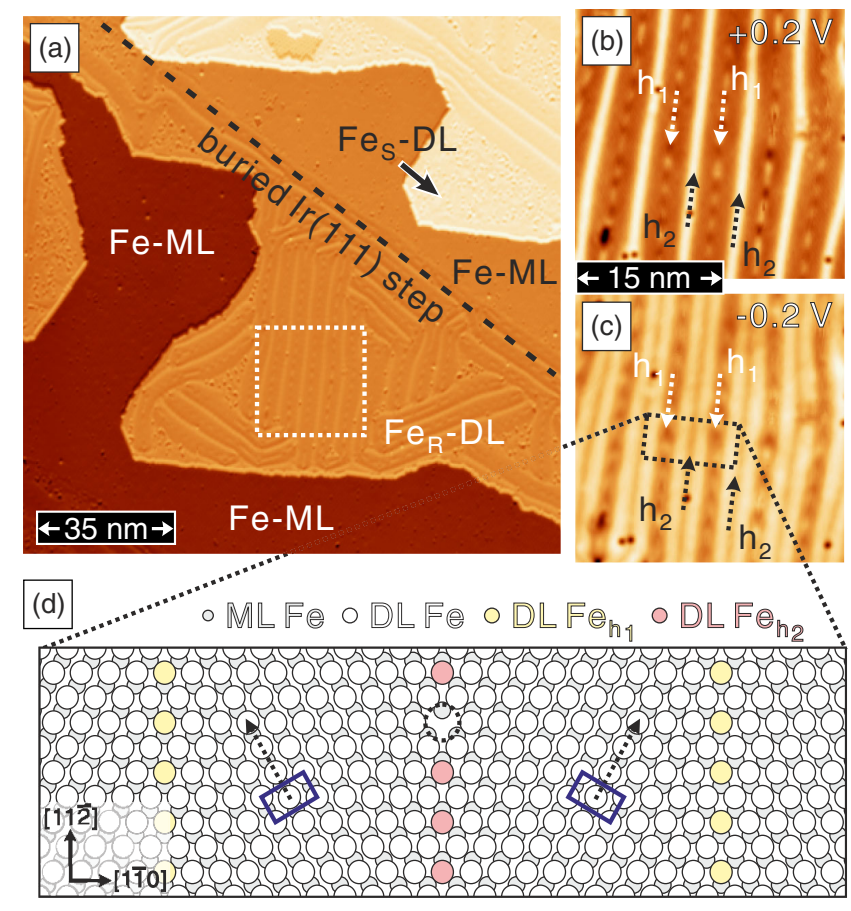

FIG. 1. (a) STM topography of about 1.6 atomic layers of Fe on an $\operatorname{Ir}(111)$ surface (measurement parameters: $U=+0.2 \mathrm{~V}, I=1 \mathrm{nA}$, $T=4.8 \mathrm{~K})$. The Fe ML grows pseudomorphically. The Fe DL consists of reconstructed $(R)$ and strained $(S)$ areas. (b),(c) Magnified topography images [see box in (a)] of a reconstructed area with periodic reconstruction lines due to uniaxial strain relief taken at $U=+0.2 \mathrm{~V}$ and $U=-0.2 \mathrm{~V}$, respectively. (d) Atomic structure model of the reconstructed Fe DL with a 5\% horizontally compressed Fe top layer on a pseudomorphic hexagonal Fe bottom layer; this locally leads to bcc(110)-like areas (blue rectangles) separated by hollow site reconstruction lines (yellow, pink). 

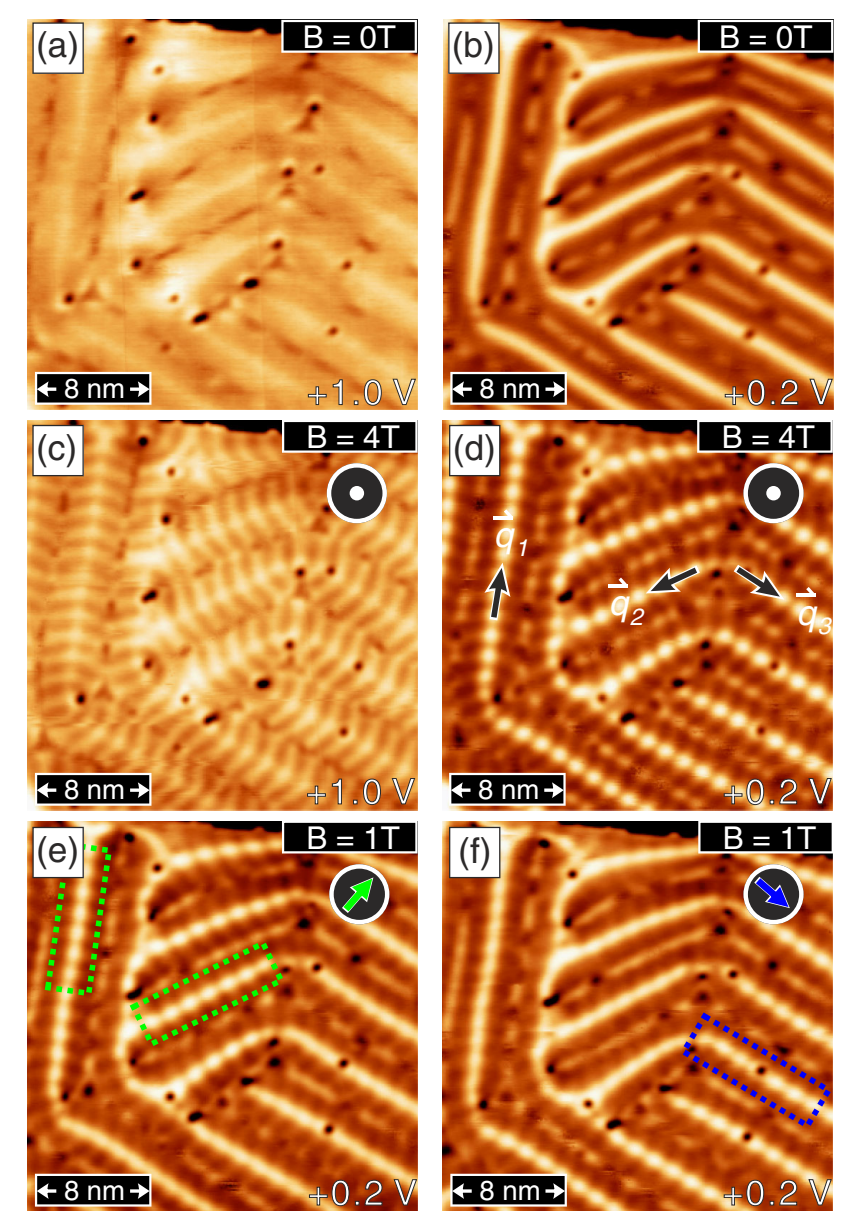

FIG. 2. (a),(b) Spin-averaged topography images of the reconstructed Fe DL with three rotational domains taken at $U=$ $+1.0 \mathrm{~V}$ and $U=+0.2 \mathrm{~V}$, respectively. The typical period of the reconstruction is $4.2 \mathrm{~nm}$, compatible with about $7 \%$ strain relief, or $16 / 15 \mathrm{Fe}$ atoms in the two Fe layers. (c),(d) SP-STM topography images performed at the same location in an outof-plane magnetic field of $4 \mathrm{~T}$ [14]. (e),(f) SP-STM topography images taken in orthogonal in-plane magnetic fields, exhibiting the respective in-plane magnetization component resulting from an alignment of the tip magnetization along the fields. The green and blue empty boxes indicate the maximum magnetic corrugation amplitudes of the different $\vec{q}$ vectors (measurement parameters: $I=1 \mathrm{nA}, T=4.8 \mathrm{~K}$ ).
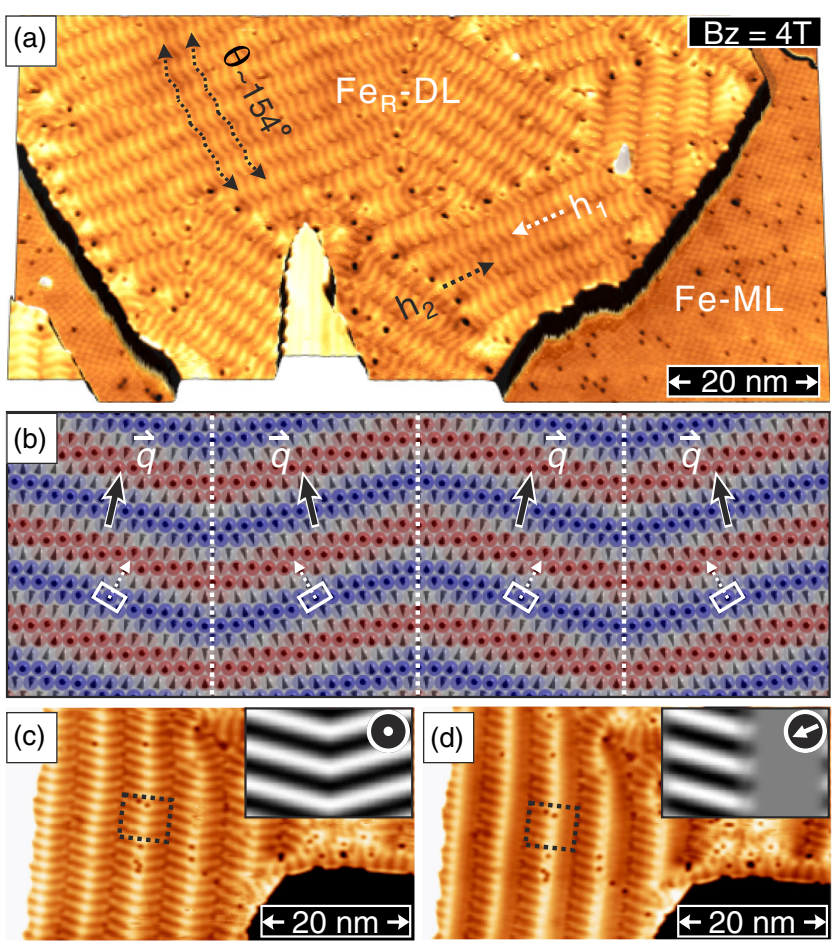

FIG. 3. (a) Overview SP-STM topography image of the spin spiral in the Fe DL and the nanoskyrmion lattice in the Fe ML; the zigzag shape of the spin spiral wave fronts with an angle of $\theta \sim 154^{\circ}$ is indicated (measurement parameters: $U=+1.0 \mathrm{~V}$, $I=1 \mathrm{nA}, T=4.8 \mathrm{~K}$ ). (b) Sketch of the magnetic state of the reconstructed Fe DL, as deduced from the SP-STM measurements. (c),(d) SP-STM topography images of the zigzag spin spiral measured with out-of-plane and in-plane spin sensitive tip, respectively. The corresponding SP-STM simulations are in the insets (measurement parameters: $U=+0.5 \mathrm{~V}, I=1 \mathrm{nA}$, $T=7.8 \mathrm{~K}, B_{z}=2.5 \mathrm{~T}$ ).

We thank Nadine Hauptmann, Daniel Wegner, and Alexander Khajetoorians for bringing the incorrect scale bars to our attention. 